\title{
COLOUR PATTERNS OF SYRPHIDAE
}

\author{
II. ERISTALIS INTRICARIUS \\ JONATHAN HEAL* \\ Department of Genetics, Liverpool University
}

Received 9.iv.79

\begin{abstract}
SUMMARY
Eristalis intricarius is a Batesian mimic of bumblebees (Bombus spp.). The thoracic pubescence may be orange or black, but a range of intermediates also occurs. The orange-haired form is recessive to the darker types of pattern (alleles $T h^{\circ r}$ and $T h^{D}$ respectively); variation within the dark-haired category seems largely influenced by environmental factors such as pupal temperature. In samples from northern parts of England there were roughly equal numbers of " orange" and "dark" morphs. These morphs are not specialised mimics of individual models, but have a good general resemblance to several species of bumblebee.
\end{abstract}

\section{INTRODUCTION}

A HAIRY hoverfly may gain some protection from predation by becoming a bumblebee mimic. Since genetic variation may arise where an edible Batesian mimic occurs with two or more suitable models (Sheppard, 1961), more than one Bombus colour pattern may be copied. Genetic polymorphisms have been examined in two Syrphid mimics of bumblebees, Volucella bombylans (Gabritchevsky, 1924) and Merodon equestris (Conn, 1972).

Eristalis intricarius L. is another variable Bombus-mimic. Verrall (1901) described a "typical " form with orange hair (" tawny pubescence ") and var. furvus with black hair, but there is also a range of intermediate forms. $E$. intricarius has a similar life history to Eristalis tenax, a honeybee mimic with genetic variation in its abdominal markings (Heal, 1979), although unlike E. tenax it overwinters as a larva. Adults of both species, feed on pollen and nectar at flowers, where mimicry of bees is both striking to an observer and useful to the fly.

This paper reports a study of the genetics of the mimetic patterns in $E$. intricarius. Although this species, in common with Volucella bombylans and Merodon equestris, uses bumblebees as models, both the extent of the variation and the way it is generated are quite different.

\section{Materials and Methods}

The breeding techniques were similar to those described for Eristalis tenax (Heal, 1979). The proportion of successful crosses was lower with $E$. intricarius but this was improved by using larger cages (at least $30 \times 25 \times 20 \mathrm{~cm}$ for a single pair) with a moderately high humidity. Since

* Present address: Department of Biological Sciences, Keele University, Keele, Staffs., ST5 5BG. 
Eristalis females oviposit in separate clusters, more than one of each sex were put into the breeding cages for some earlier crosses-as was done satisfactorily for $E$. tenax-but this was discontinued later. Broods judged to be from a single mating are added together in table 2. In suitable conditions females often laid their first batch of eggs within 14 days of emergence. Each batch was transferred to a larval medium of rotting grass or rabbit droppings mixed with water. The main difficulty with this species is that larvae from autumn broods may diapause, even when kept at over $20^{\circ} \mathrm{C}$. When no larval diapause occurred, the time from oviposition to adult emergence was about 30 days at $18-20^{\circ} \mathrm{C}$.

\section{Colour patterns}

Adult Eristalis intricarius were classified according to the colour of the dorsal thoracic pubescence. Five arbitrary categories were distinguished (fig. 1) but there was virtually a continuous series between the extremes:

T ("typical ") Orange-haired thorax (possibly yellow in faded specimens), apart from a few black hairs along the post-alar calli.

TB Mainly orange, but with patches of black at the sides of the rear part of the thorax.

B (banded) Median orange stripe, but black at both sides.

FB Mainly black, but with some patches of orange or yellow amongst the black in the middle of the thorax.

F (furvus) Black-haired thorax.

The abdomen of male $E$. intricarius is covered mainly with orange pubescence but this may be infiltrated by numerous black hairs in the darker forms. The females have a white or pale yellow tail, with a broad black band in front. The $\mathrm{T}$ female also has a patch of orange hairs on the anterior part of tergite 2 .

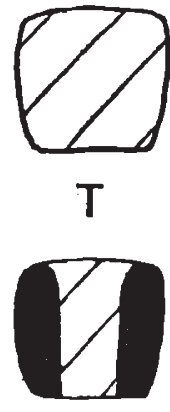

B

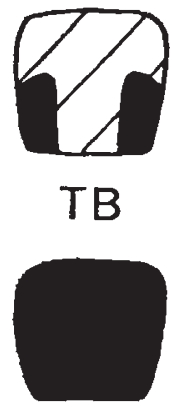

F

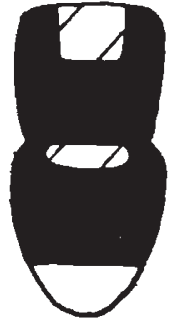

FBq

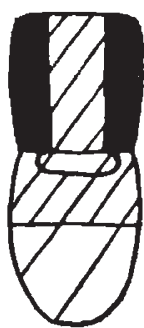

FBơ

Fig. 1.-Colour patterns of Eristalis intricarius: classification into five grades from the thoracic pubescence. For type FB, examples of a male and a female illustrate the usual sex difference in this grade; these also show the sexual dimorphism in colouration of the abdominal pubescence, although the amount of black varies with the thoracic pattern. Unshaded $=$ white hair. Wide hatching $=$ orange or yellow. Close hatching $\times$ mixture of black and orange/yellow. Heavy shading = black. 


\section{REsults}

\section{(i) Genetic variation}

The broods reared from captured females (table 1) and from laboratory matings (table 2) show that "orange-hair" (i.e. grade $\mathrm{T}$ only) is recessive to "dark-hair" (which comprises grades F, FB, B and TB). This locus for thoracic hair colour will be termed $T h$, with alleles $T h^{o r}$ (orange) and $T h^{D}$ (dark).

Orange females yield either entirely orange-haired progeny (I74/6, 175/58, I75/70) or broods segregating 1:1 (I75/78). The former occurs if both parents are homozygous for the orange allele (e.g. Iod in table 2).

Dark-haired Eristalis intricarius may be homozygous $\left(T h^{D} T h^{D}\right)$ or heterozygous $\left(T h^{\text {or }} T h^{D}\right.$ ); the latter is indicated for flies in $1: 1$ broods. Matings of orange $\times$ heterozygous dark produced the $1: 1$ broods in table 2(ii). The offspring of cross Ioj (table 2(iii)) showed a close approximation to the $3: 1$ ratio expected from two heterozygotes. Most of the other dark $\times$ dark crosses suggested the same type of mating but the darkhaired brood from Io $\delta$ indicates that at least one parent was homozygous $T h^{D} T h^{D}$. Crosses Io $\varepsilon, \mathrm{Ija}$ and $\mathrm{Ij}$ s show that the TB females tested, although phenotypically very close in appearance to $\mathrm{T}$ females, had a heterozygous genotype.

The dark-haired category was clearly more variable than the orange morph, but this need not imply genotypic variation beyond the presence of heterozygotes and homozygotes. To test for this, the darker grades were scored as: $\mathrm{F}=1, \mathrm{FB}=2, \mathrm{~B}=3, \mathrm{~TB}=4$. For each brood the midparent value $(\bar{p})$ and mean values for male $(\bar{m})$ and female $(\bar{f})$ offspring were calculated. Orange-haired offspring were disregarded. For matings between pairs of dark-haired flies, the regression coefficient of $\bar{o}$ (mean offspring value $=\frac{1}{2}(\bar{m}+\bar{f})$ ) on $\bar{p}$ is an estimate of the heritability of colour patterns within the dark-haired category. From the data in table 2(iii) (laboratory matings only):

$$
b_{\bar{a} / \bar{p}}=-0.46 \pm 0.17
$$

Therefore there is no evidence of any genetic variability other than in the segregation of $T h^{o r}$ and $T h^{D}$ alleles. Environmental factors are indicated by a "heritability" of less than zero. However, this estimate is based on broods derived from very few wild females and so further genetic variation in natural populations is still a possibility.

\section{(ii) Environmental variation}

There is evidence that pupal temperature can influence the hair colour of the adult (table 3). The dark-haired morph seemed very sensitive to differences in pupal incubation temperature. At $25^{\circ} \mathrm{C}$ many $\mathrm{TB}$ and $\mathrm{B}$ flies were produced, whilst below $18^{\circ} \mathrm{C}$ a high proportion were of the darkest grade, entirely black-haired on the thorax. Therefore, warmer conditions yield intermediate patterns that are absent at low temperatures. A change of temperature before all the adults of a brood have emerged from the pupae can thus produce a misleading bimodality within the dark group (see Ija2 in table 3). However, some further explanation seems to be required 

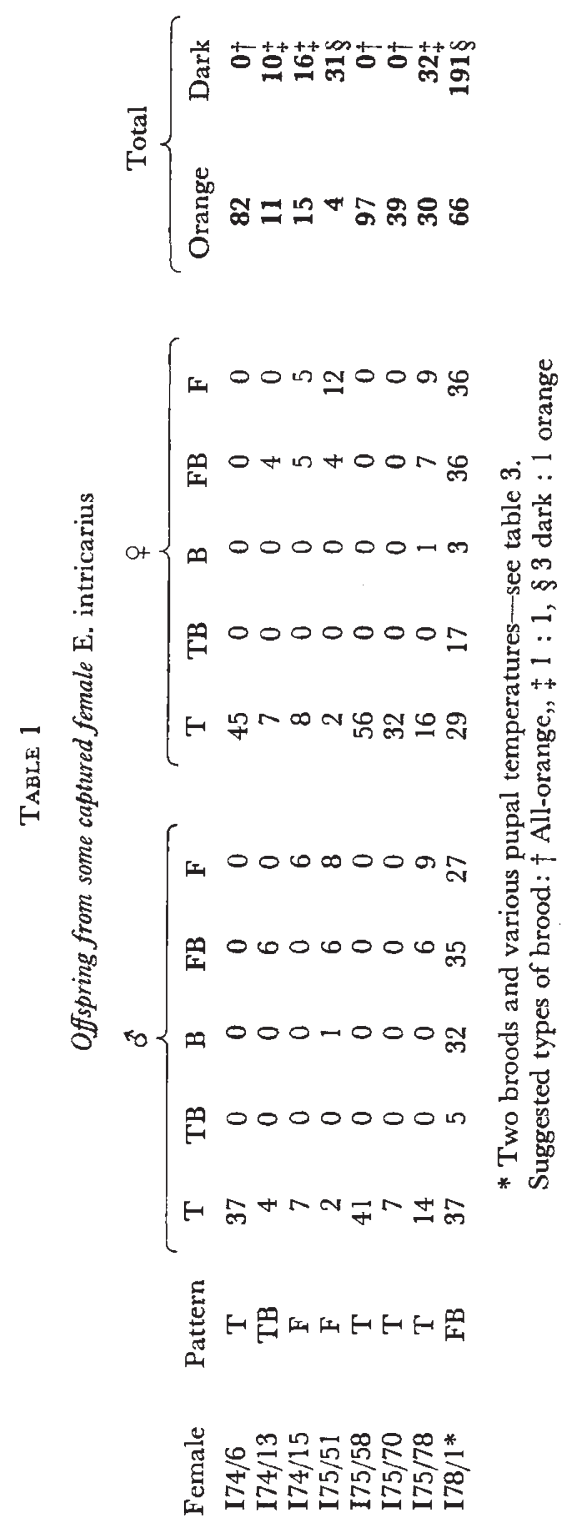


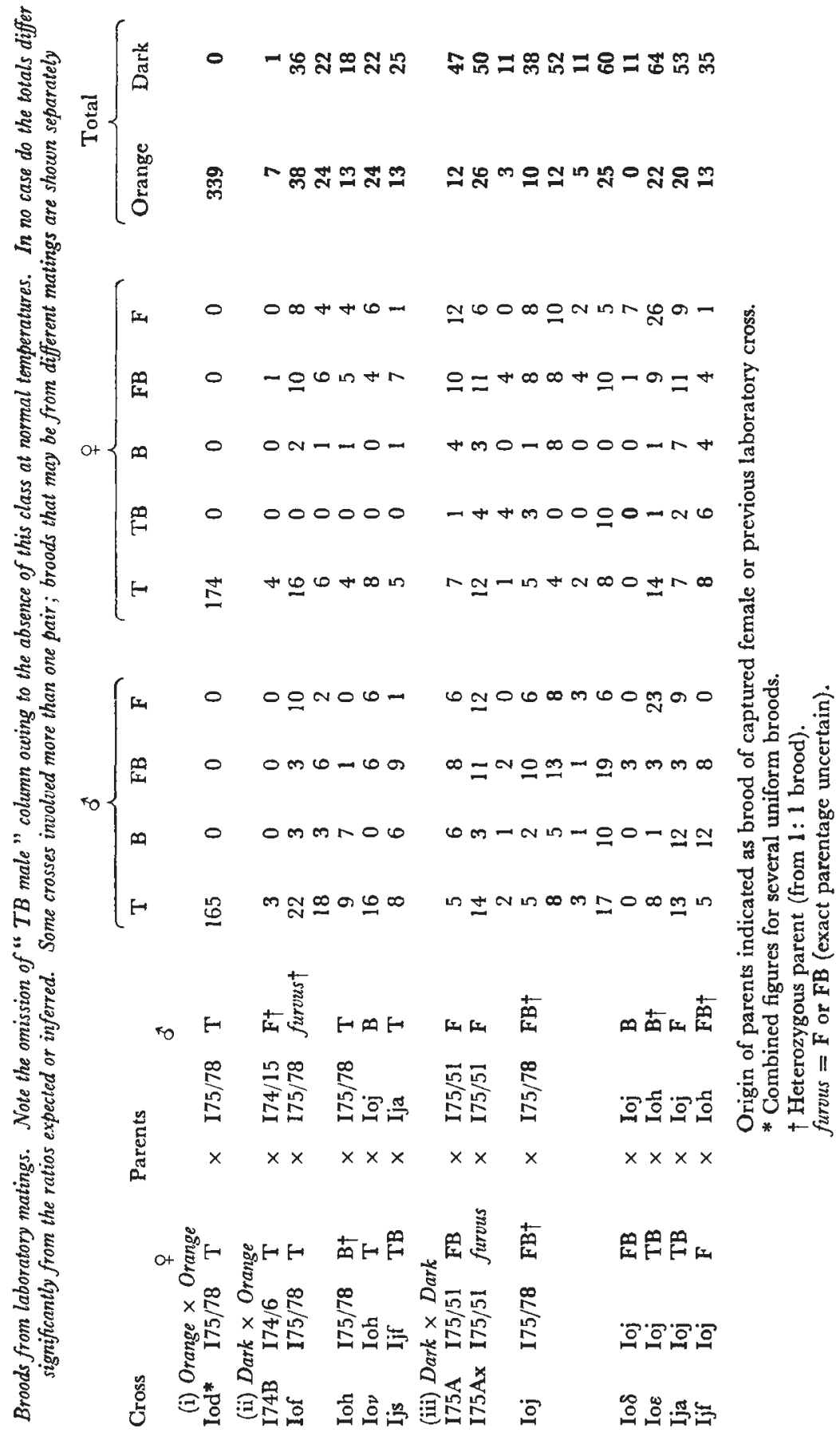


竞

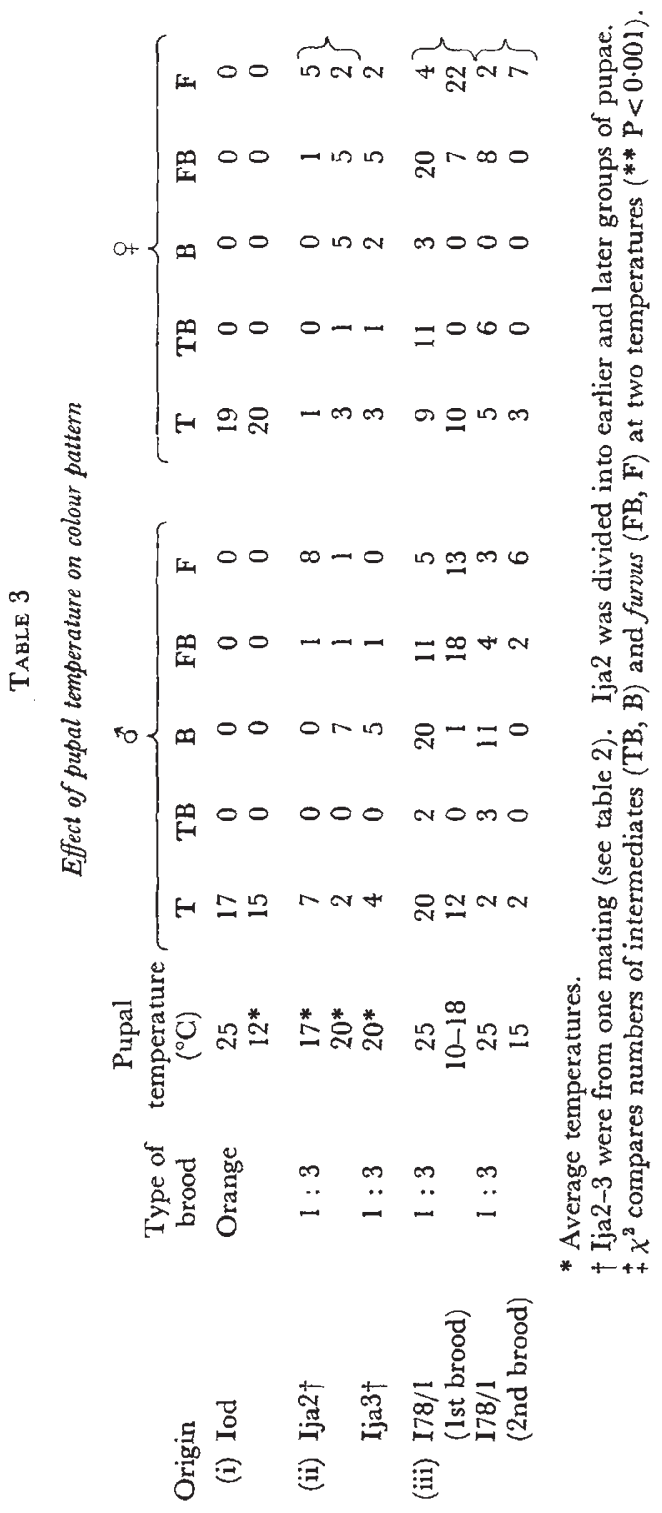


for the phenotypic variation amongst "dark-haired" $E$. intricarius reared under very similar conditions (e.g. broods of $178 / 1$ from pupae at $25^{\circ} \mathrm{C}$ ). The rarity of $\mathrm{TB}$ males, even in warmer conditions, suggests a sex difference in response to higher temperatures; this is not unexpected since the abdominal colourations of males and females differ appreciably (i.e. there is not selection for exactly the same patterns in both sexes).

The negative regression coefficient for the parent-offspring comparison (above) probably resulted from seasonal changes in the temperature of the room in which pupae were kept, so that successive generations experienced dissimilar conditions. The broods with the highest proportion of extreme phenotypes (Io $\delta$, Io $\varepsilon$, Iov) emerged in January and February, when the average laboratory temperature fell below $17^{\circ} \mathrm{G}$. However, a wide range of temperatures showed no effect on the colouration in an all-orange brood (Iod). Both the extent and the shade of the orange pubescence were very uniform through all the conditions tested.

\section{(iii) Patterns in natural populations}

Only limited samples were taken because $E$. intricarius rarely attains the local abundance noted in several other Eristalis species. Some were collected from rural areas in north Cheshire (mainly near Neston, Wirral) and north Staffordshire (the campus of Keele University), but very few were seen at urban sites in Liverpool where other Eristalis species were sampled regularly. Most $E$. intricarius were found between mid-June and mid-August, and they were usually captured when visiting flowers (e.g. Rubus fruticosus, Centaurea nigra, Senecio jacobaea, Heracleum sphondylium).

There were 48 per cent of the orange-haired morph in the Cheshire samples, and 38 per cent in north Staffordshire (see table 4). These figures correspond to estimates of 0.7 and 0.6 for the frequency of the $T h^{\text {or }}$ allele, assuming Hardy-Weinberg ratios. Observations at Keele in 1977-78, of flies which eluded capture, gave further totals of 24 orange and 21 dark. Thus in these two localities the two morphs occur in roughly equal numbers.

\section{(iv) Edibility}

Eristalis intricarius seems to be quite palatable to birds. Several were eaten by a captive robin, and by a pair of wild great tits which had been trained to visit a window ledge where food was left. The robin dealt with the $E$. intricarius offered to it rather cautiously at first, pecking and rubbing the flies on the ground, but later ones were eaten quite rapidly. It seems probable that the initial caution was due to the appearance of the flies, and this was overcome when they proved to be harmless and edible. Other Eristalis species were also eaten $(E$. tenax, $E$. arbustorum, $E$. nemorum, $E$. pertinax). Therefore all of these are Batesian mimics of bees.

\section{Discussion}

A mimetic polymorphism may involve two or more discrete varieties without intermediates. Volucella bombylans, another Syrphid mimic of bumblebees, has two principal morphs in Britain: bombylans, a mimic of redtailed bees and plumata, which is yellow, black and white. The data of 


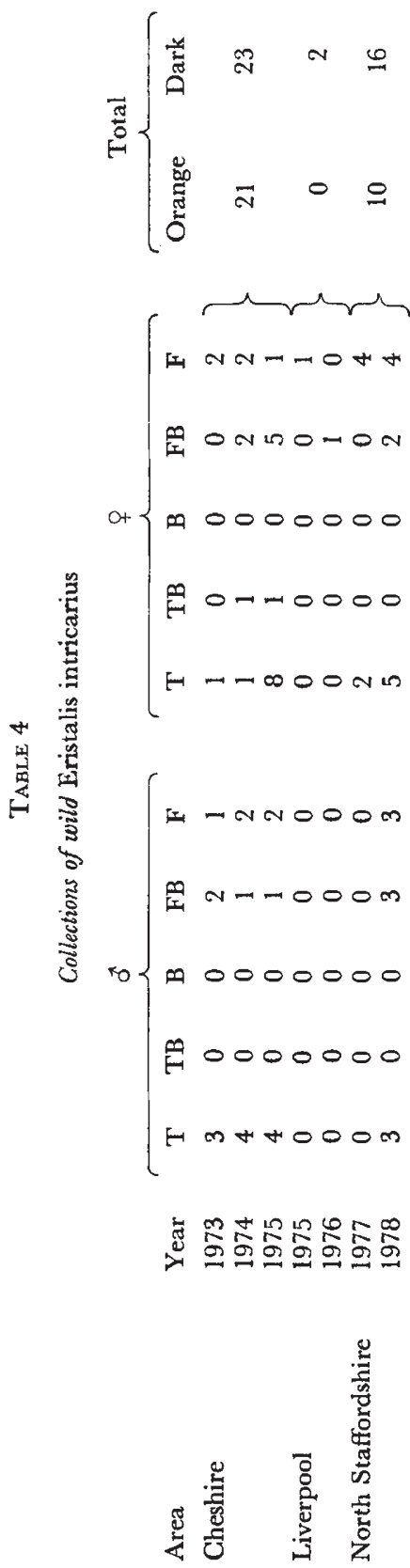


Gabritchevsky (1924) indicate that the bombylans pattern is dominant. The Volucella larva is a scavenger in bee nests and so this obligate association of model and mimic may have led to selection for precise mimicry. In the narcissus bulb fly, Merodon equestris, there are several loci producing an array of colour patterns, which may be good, moderate or poorer mimics of various Bombus species (Conn, 1972).

Eristalis intricarius of any colour pattern look like small bumblebees. Darker grades of female (B, FB, F) resemble four species of striped Bombus that are common in Britain. A light band usually occurs across the middle of the body due to yellow hairs on the scutellum. The tail may be white (as in $B$. lucorum and $B$. hortorum), yellowish (as in B. terrestris), but not red as in $B$. pratorum. The black and red $\boldsymbol{B}$. lapidarius is mimicked by furvus males having reddish-orange hairs on the rear part of the abdomen. The mainly orange bee $B$. agrorum Fab. (pascuorum Scop.) is a model for the brighter grades of $\boldsymbol{E}$. intricarius, both male and female. Orange-haired males also resemble quite closely females of the sexually dimorphic mining bee Andrena armata ( fulva), although this may be incidental.

However in no case is there really " perfect" mimicry of one particular model, but rather a good bee-like appearance which is more specific for the extreme forms and more general for the intermediates. At a distance, $E$. intricarius appear less conspicuously coloured than many bumblebee workers, partly because the fly's pubescence is shorter and less dense. The fine and unbranched thoracic hairs stand up vertically rather than lying back over the cuticle, and so the colouration is more striking when viewed from the side.

Genetically, the colour patterns should be divided into an orange-haired morph, which varies little, and a rather variable dark morph, the lighest grade of which (TB) differs only slightly from the orange-haired phenotype (T). Dark is dominant to orange, yet it appears that the amount of black hair on the " dark-haired" flies is influenced by environmental factors such as pupal temperature. Thus one would predict seasonal fluctuations in the amount of phenotypic variation, but at present my own samples are too small to demonstrate this.

In Britain, several hoverflies that are bumblebee mimics (including Volucella bombylans and Merodon equestris) have a single generation per year which appears in June while the numbers of Bombus workers are increasing. Conn (1976) has suggested that other mimetic species flying with $M$. equestris during the shortened flight season in the Pyrenees are a cause of the restricted polymorphism that he observed there; absence of variation at the bulborum locus limited the number of possible phenotypes. Thus both a close ecological association with the species copied (as in Volucella) and the presence of other mimics may influence the precision and diversity of the mimetic patterns.

Selection for specialised mimicry could be reduced in Eristalis intricarius because of a longer flight period with two generations each year. The small spring generation, from larvae that were in diapause over winter, appears before Bombus workers are active. The more numerous second generation occurs in late summer when bumblebees having various types of colouration are foraging. Although there are similarities between several species of Bombus, and also Psithyrus (cuckoo-bees), the Müllerian mimicry is less extreme than amongst wasps. Predators could therefore acquire, by the 
end of summer, a generalised aversion to any bee-like form. However it is not obvious to what extent the relative advantages of different colour patterns would vary during the year in $E$. intricarius. Intermediates were, in any case, infrequent in the samples taken and so it is yet to be shown how the responses to rearing temperature are adaptive.

Acknowledgments.-Most of this work was done with the aid of an SRC studentship at the Genetics Department, Liverpool. I am very grateful for the critical supervision of the late Professor P. M. Sheppard.

\section{REFERENGES}

CONN, D. L. T. 1972. The genetics of mimetic colour polymorphism in the large narcissus bulb fly, Merodon equestris Fab. (Diptera : Syrphidae). Phil. Trans. R. Soc. B., 264, 353-402.

CONN, D. L. T. 1976. Evidence of restricted mimetic colour polymorphism in the large narcissus bulb fly, Merodon equestris Fab. (Diptera : Syrphidae) in the Pyrenees. Heredity, 36, 185-189.

GABRITCHEVSKY, E. 1924. Farbenpolymorphismus und Vererbung mimetischer Varietäten der Fliege Volucella bombylans und anderer "hummelähnlicher" Zweiflügler. Z. Indukt. Abstamm. u. Vererb., 32, 321-353.

HEAL, J. R. 1979. Colour patterns of Syrphidae. I. Genetic variation in the dronefly Eristalis tenax. Heredity, 42, 223-236.

SHEPPARD, P. M. 1961. Recent genetical work on polymorphic mimetic Papilios. In Insect Polymorphism (ed J. S. Kennedy), pp. 20-29. Royal Ent. Soc., London.

verrall, G. H. 1901. British Flies: Platypezidae, Pipunculidae and Syrphidae. Gurney and Jackson, London. 